\title{
Diabetic Retinopathy Pathological Signs Detection using Image Enhancement Technique and Deep Learning
}

\author{
Abdul Hafiz Abu Samah*, Fadzil Ahmad, Muhammad Khusairi Osman, Noritawati Md Tahir, \\ Mohaiyedin Idris, and Nor Azimah Abd. Aziz.
}

\begin{abstract}
The screening of diabetic retinopathy (DR) affects the visual inspection of retina images taken by ophthalmologists to detect the specific signs of pathology such as exudate, hemorrhage (HEM) and microaneurysm (MA). However, this process is currently conducted manually in many hospitals. Therefore, it is time-wasting and risky for humans to make mistake. In general, this paper introduces an automated machine learning algorithm for detecting diabetic retinopathy (DR) in fundus images. It also involves an image pre-processing enhancement technique to support accuracy on deep learning for DR classification. For the image enhancement process, high-pass filter, histogram equalization and de-haze algorithm are applied to improve the visual quality of fundus images. By using four convolution layers, a CNN architecture is set up to classify the three pathological signs; HEM, MA and exudate. Two public online datasets, eOphtha and DIARETDB1 are used to evaluate the performance of this system. From training and testing results using enhanced DR images, a slight improvement in classification accuracy is revealed, compared to those original images with no enhancement for both datasets.
\end{abstract}

Index Terms - Diabetic retinopathy, microaneurysm, hemorrhage, exudate, convolutional neural network, high pass filter, histogram equalization, de-haze.

\section{INTRODUCTION}

D IABETES is a major disease that directly affects 422 million people worldwide. World Health Organization (WHO) estimates that Malaysia will have 2.48 million people with diabetes in 2030 [1]. Diabetes is caused by the pancreas failure to generate insulin in the human body [2]. Eventually, this disease can cause organ failure. One of the major concerns of diabetes is when it starts affecting the retina of the eye. This disease is called diabetic retinopathy (DR). In addition, late treatment at an early stage and unsupervised DR can cause vision loss. 2021

This manuscript is submitted on $10^{\text {th }}$ May 2020 and accepted on $15^{\text {th }} \mathrm{Jan}$

Abdul Hafiz Abu Samah, Fadzil Ahmad, Muhammad Khusairi Osman, Noritawati Md Tahir, Mohaiyedin Idris, are with the Faculty of Electrical Engineering, University Teknologi MARA, 40450 Shah Alam, Selangor (email: hafiz_mppuitmpp@yahoo.com).

Nor Azimah Abd. Aziz is with the Faculty of Medicine, Universiti Teknologi MARA

1985-5389/C 2021 The Authors. Published by UiTM Press. This is an open access article under the CC BY-NC-ND license (http://creativecommons.org/ licenses/by-nc-nd/4.0/).
However, early diagnosis and treatment will help save more costs and prevent patient from loss-sightedness [3].

A traditional DR examination would be based on a manual examination using ophthalmoscopy methods based on retina. However, these conventional methods are time consuming, tedious, and can lead to inconsistent diagnosis [2]. From the past three decades, the automatic diagnosis of DR using computer aid diagnosis (CAD) techniques has become popular among medical researchers. This helps reduce the workload of ophthalmologists and provide timely diagnosis for early treatment[2]. For automated screening, there are several machine learning techniques that have been proposed from previous research to detect and screen common signs of DR pathology such as microaneurysm (MA), hemorrhage (HEM), and exudates [4]. These frameworks include the use of artificial neural network (ANN) [5], support vector machine (SVM) [6], k-nearest neighbor (K-NN) [7] and convolution neural network (CNN) [4], [8]-[10].

Lately, the deep learning in DR screening and detection systems using CNN has shown an increasing trend among researchers. $\mathrm{CNN}$ is the most method using deep neural network learning algorithms for image identification. CNN involves minimum pre-processing as it automatically learns the image features based on the input image given compared to the traditional algorithm method which needs to manually extract the feature. This technology helps human effort and its ability to solve complex feature designs is the main key advantage.

Inspired by the work [8], this paper introduces a deep learning algorithm using the convolution neural network (CNN) for an automatic detection of diabetic retinopathy (DR) in fundus images. However, previous works done by researchers use histogram equalization as contrast enhancement in the image pre-processing stage on the DR image. The new image enhancement technique proposed in this paper seeks to compare the performance of the DR classification using CNN.

\section{Clinical Features}

As the diagnosis of DR disease, specific DR features need to be screened out by an ophthalmologist from a suspected fundus picture of DR patients. These pathological features include MA, HEM and exudates as shown in Fig. 1. 


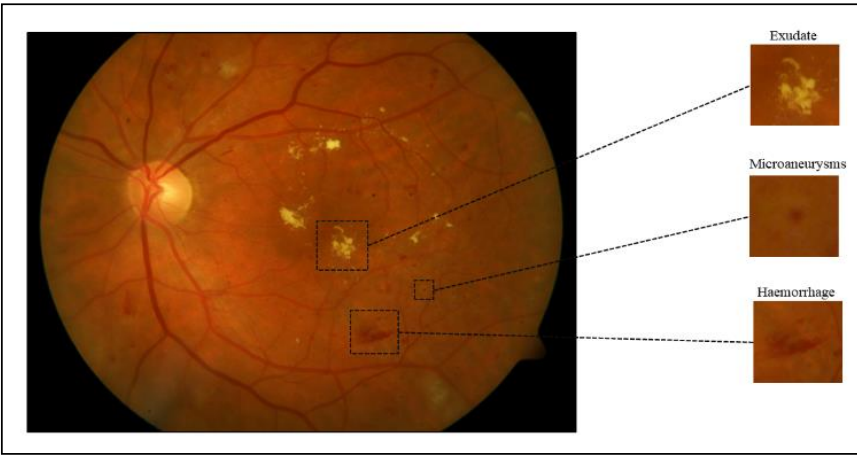

Fig. 1. Three (3) common DR pathological signs.

Firstly, MA is usually a small round shape, dots and dark red spots caused by high blood sugar (hyperglycemia) in the blood vessel wall [11]. The amount of MA increases as the DR increases. The MA shape dimension range is between $20 \mathrm{um}$ and 200um equivalent to 1-3 pixels in different images [11].

Secondly, HEM can appear due to the leakage or rupture of blood from the capillaries or blood vessels. It looks like red flames, dots and spots [12]. Usually HEM is $125 \mu \mathrm{m}$ larger in size than MA [13]. Lastly, exudate usually appears in bright white or yellowish white with a sharp edge to the bloodstream background [11]. It is from the lipoproteins coming from the retinal tract to the outer layer of the retina. Exudates are often arranged in a circular shape [13].

\section{METHODOLOGY}

\section{A. Data Collection}

In this research, two (2) datasets were used namely DIARETDB1 [15] and e-Ophtha [14].

DIARETDB1: Contains a total of 89 fundus images of which 84 types of mild non-proliferative signs (Microaneurysms) of diabetes retinopathy, and 5 normal normal images with no signs of diabetes retinopathy are observed, according to all experts participating in the assessment.

$e$-Ophtha: E-ophtha coming from two subsets of a dataset: Firstly, e-ophtha-MA dataset totalling 148 images with microaneurism (MA) or minor HEM. Secondly, the dataset is e-ophtha-EX containing 47 pictures with exudates (EX).

This dataset is given the authorization or reference (ground truth) to the MA and Exudate marks. This basic truth description is provided by two experts in each department of ophthalmology. Table I shows the number of normal and abnormal images in various databases.

TABLE I

TOTAL NUMBER OF ABNORMAL AND NORMAL FUNDUS IMAGE FOR DIARETDB1 AND E-OPTHA

\begin{tabular}{|lll|}
\hline Dataset & \multicolumn{2}{l|}{ No. of Image } \\
\cline { 2 - 3 } & Normal & Abnormal \\
\hline & & \\
DIARETDB1 & 5 & 84 \\
e-Ophtha-MA & 233 & 148 \\
e-Ophtha-Ex & 35 & 47 \\
\hline
\end{tabular}

\section{B. Image Enhancement}

One of the problematic pictures of retina is that there are variations in the brightness and contrast that make it difficult to identify DR features possibly resulting from the bad performance of deep neural network. Moreover, each design has its own uniqueness [11]. There are several reasons why the fundus images are blurry and the images are in low lights when taken by ophthalmologists. One of the common reasons is the inability to open the pupil (constrict) under bright light condition. A cloudy aging cataract at the eye lens also provides less light to reach the retina. Because of the inconsistent brightness and contrast colour of the retinal image, to achieve the objective f getting a strong $\mathrm{CNN}$ performance, our proposed method is to enhance all the dataset images using the high pass filter, histogram equalization and de-haze algorithm technique to remove the noise.

High-pass filter is widely used in the biomedical image enhancement. This filter attenuates low frequency (unwanted noise) and allows high frequency to pass filter. As the result, the high frequency area will result in sharpening [15]. Now, the image processing uses the sharpened image as ridge and edge detection. High pass filter was set by the frequencies outside radius $D_{0}$. The equation of this high pass filter comes with the number of order $n$ with distance $\mathrm{D}(\mathrm{u}, \mathrm{v})$ and a cut-off frequency $\mathrm{D}_{0}$ which is defined as:

$$
H(u, v)=\frac{1}{1+\left[D_{0} / \mathrm{D}(\mathrm{u}, \mathrm{v})\right]^{2 n}}
$$

However, applying the high-pass filter on this fundus image will also enlarge the intensity gradients in the images. As the result, it affects the colour intensities of the images which make RGB colour look faded. Therefore, the histogram equalization (HE) has been used to enhance the image and to visualize the pathological signs. Especially in the medical domain, the HE technique enhancement of image is used in a wide range of medical imaging applications [16]-[18]. The image contrast improves the image by flattening and stretching the dynamic range of the histogram for the target image [19]. Assuming the digital image, $F(i, j)$, with total $\mathrm{N}$ pixels and grey level range $[0$, $\mathrm{K}-1$, the density function image was calculated based on mathematical equation (2):

$$
p(k)=\frac{n_{k}}{N}, \quad \text { for } k=0,1, \ldots, K-1
$$

Where $n_{k}$ is the number of pixels with the number of grayscale $k$ in the image. Next, the cumulative distribution function $(\mathrm{CDF})$ of the image $F(i, j)$ can be derived by (3):

$$
C(k)=\sum_{m=0}^{k} P_{m}, \quad \text { for } k=0,1, \ldots, K-1
$$

From the CDF value in equation (3), HE matches an input level $k$ to the output level $H_{k}$ as described in equation (4):

$$
H_{k}=(K-1) \cdot C_{k}
$$

Therefore, the gain H_k at the output level for HE can be referred to in Formula (5): 


$$
\Delta \mathrm{H}_{k}=H_{k}-\left(H_{k}-1\right)=(K-1) \cdot P(k)
$$

From the related equations, it is proven that the increase in the level of gain $H_{k}$ is proportional to the probability of the associated level $k$ in the original image [20]. HE helps to figure out the hidden details within the image by stretching out the contrast of the local region. Finally HE helps to make observable pathological signs within the region processed.

Besides the fact that the histogram equalization applies the contrast enhancement, the other method is used to enhance the low light image especially for retinal images with poor lighting condition. The de-haze algorithm on the inverted video was applied by [21], taking this method approach to be applied to the fundus image.

For images in hazy condition, the intensities of the background pixels are always high in all the colour channels. However, usually there is at least one colour which is low in the channel because of the shadow, shape, colour and etc [21]. First of all, the De-haze enhancement algorithm is proposed by applying an invert operation on the low image. The invert model is described using:

$$
R^{c}(x)=255-I^{c}(x),
$$

where $c$ is the RGB color channel. $I^{c}(\mathrm{x})$ is the intensity of low lighting input image colour channel of pixel $\mathrm{x}$. Therefore $R^{c}(x)$ is the intensity of the inverted image $\mathrm{R}$. The image haze removal model is shown in (7):

$$
R(x)=J(x) t(x)+A(1-t(x))
$$

where $\mathrm{A}$ is the global atmospheric light. $R(x)$ is the intensity of pixel $\mathrm{x}$ from the camera. $J(x)$ is the intensity of the original object. $t(x)$ represents the percentage of the light emitted from the object. The critical part to remove the haze in this algorithm is to estimate $\mathrm{A}$ and $t(x)$ from the input image $I(x)$. As the result it can recover $J(x)$ from $I(x)$. Since the atmosphere is homogenous, the $t(x)$ can be expressed as follows :

$$
t(x)=e^{-\beta d(x)},
$$

Where $\beta$ is the scattering coefficient of the atmosphere. It indicates that the scene radiance is attenuated exponentially with the scene depth $d$ [22]. Thus, according to equation (7), the recovery of $J(x)$ can be expressed as follows:

$$
J(x)=\frac{R(x)-A}{t(x)}+A,
$$

However, equation (9) may lead to the under enhancement of the low -lighting image. To optimize the calculation of $t(x)$, the focus is to enchance the region of interest such as MA, HEM, and Exudate while avoiding the processing for the background (no-sign) in low light images. To optimize $t(x)$, the multiplier $P(x)$ is introduced into (9), and the $\mathrm{P}(\mathrm{x})$ can be expressed as follows:

$$
P(x)=\left\{\begin{aligned}
2 t(x), & 0<x<0.5 \\
1, & 0.5<x<1
\end{aligned}\right.
$$

Therefore, the new recovery image equation becomes

$$
J(x)=\frac{R(x)-A}{P(X) t(x)}+A,
$$

When the $t(x)$ is smaller than 0.5 , the pixel needs to be boosted. Thus, a small value is assigned $P(x)$ to make $\mathrm{P}(\mathrm{x}) \mathrm{t}(\mathrm{x})$ smaller in order to increase the RGB intensities of the pixel.

For low-lighting images, once $\mathrm{J}(\mathrm{x})$ is obtained, the image applies step equation (6) to re-invent the back image to produce the enhanced image $\mathrm{E}$ from the original input image. In rhe overall method of the enhancement image process flow, Fig. 2 represents the stage of enhancement flow of the two different proposed methods.

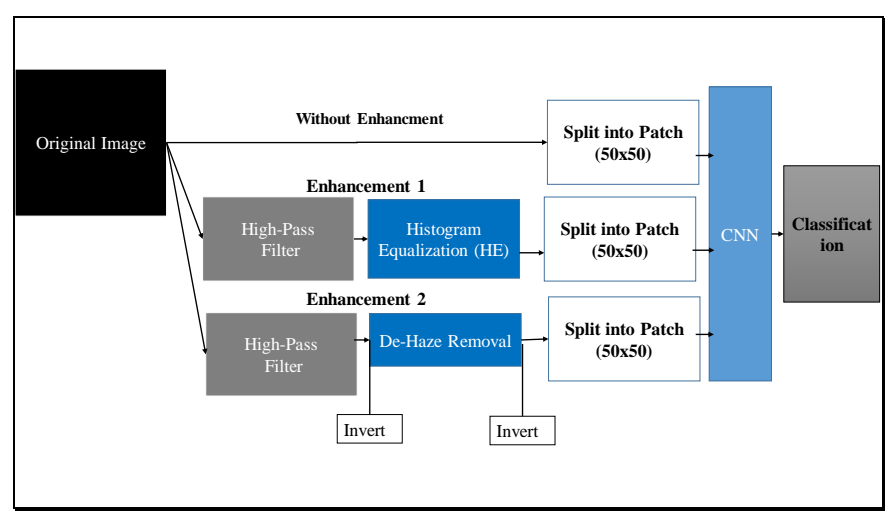

Fig. 2 Enhancement stage within flow of the proposed approach.

\section{Patch split and labeling}

After the image pre-processing process has been completed, the image is cut into patches with the dimension of 50x50 pixel. This size of patch is defined based on the sufficient area to cover all the categories of pathological signs in fundus images. These patches are labelled and sorted into the associated groups.

Total patch images are categorised by comparing the original image with the ground truth images. The patch will automatically be compared and sorted in class based on the three pathological signs : exudate, HEM, MA and background (no signs of DR) as shown in Fig. 3. Patch to patch is automatically compared based on the ground truth and finally sorted into class as shown in Fig.4. The data image augmentation like the duplicate and rotation was applied on the patches to increase more dataset training and testing to improve the performance of $\mathrm{CNN}$ for classification.

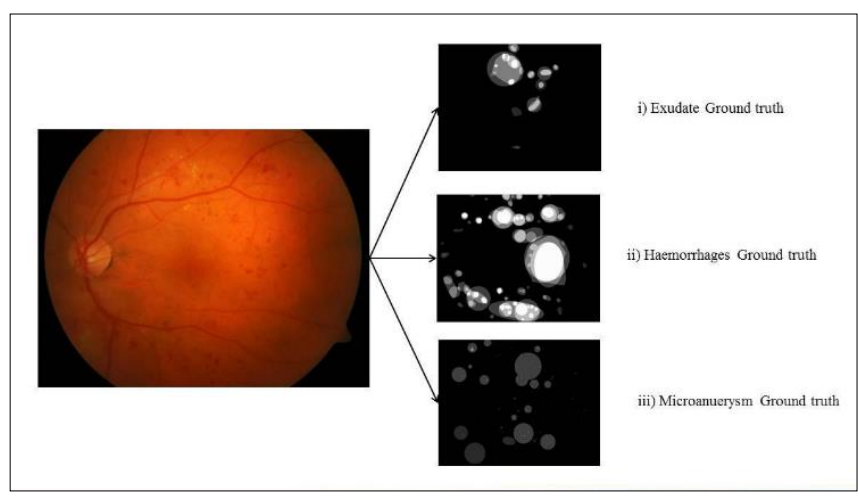

Fig. 3. Image follow the ground truth to identify position each sign category before split. 


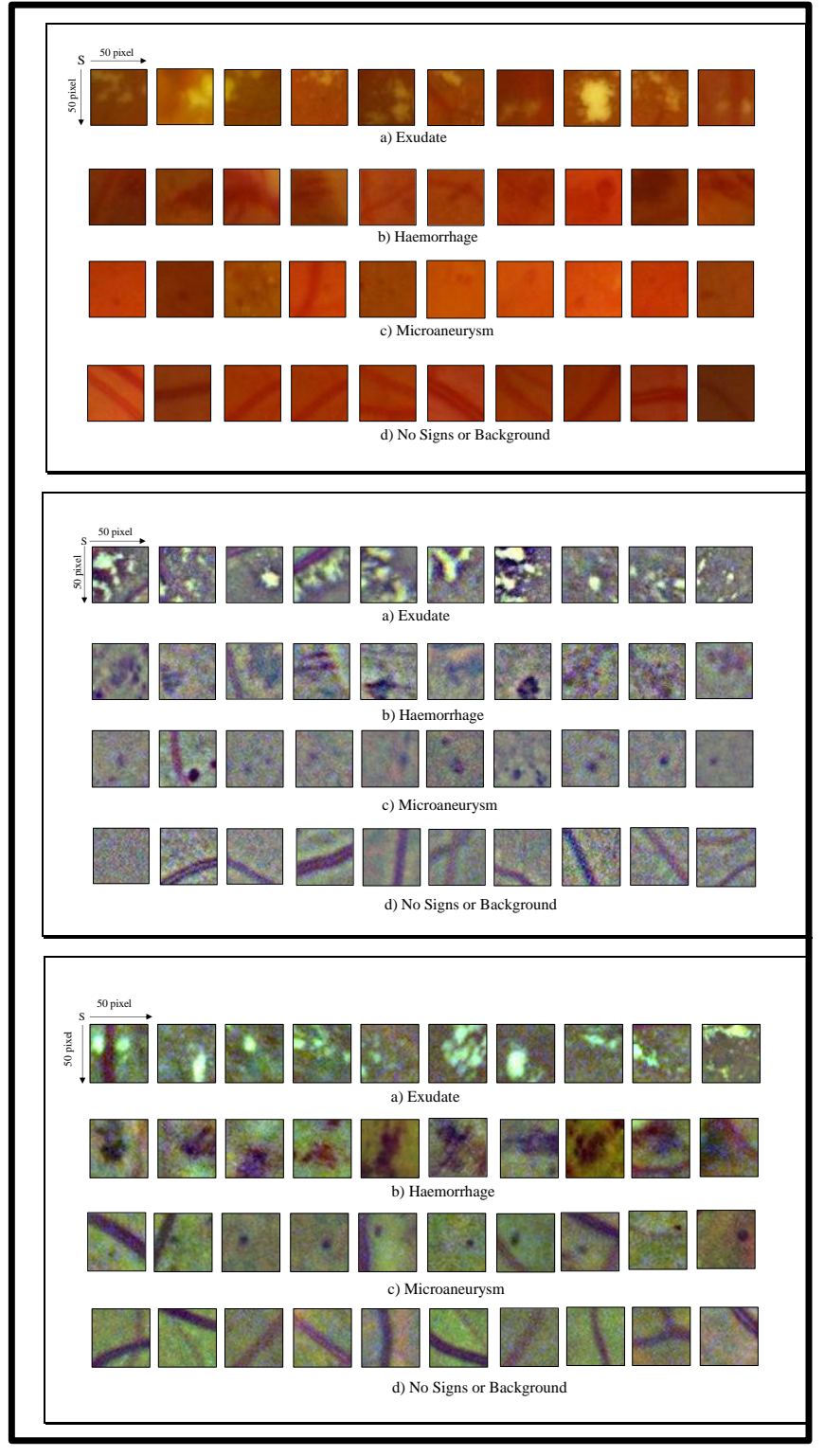

Fig. 4. Patches are grouped into MA, HEM, exudate and no-Sign.

A total of 5569, 3328, 4816 patches for MA, HEM, exudate and 6642 patches with no pathological sign (background) for DIARETDB1 were produced. Meanwhile, a total of 2292, 4193 patches for MA, exudate and 6863 patches with no signs (background) were produced for the e-Optha dataset. The total number patch was divided into $60 \%$ for training and remaining $40 \%$ used for the testing validation purpose as shown in Table II and Table III:

TABLE II.

TOTAL NUMBER PATCHES OF DIARETDB1

\begin{tabular}{|l|l|l|l|l|}
\hline Type of Dataset & Microaneurysm & Haemorrhage & Exudate & $\begin{array}{l}\text { No- } \\
\text { Sign }\end{array}$ \\
\hline Training & 3342 & 1998 & 2891 & 3987 \\
\hline Testing & 2229 & 1332 & 1927 & 2657 \\
\hline Total Number & 5571 & 3330 & 4818 & 6644 \\
\hline
\end{tabular}

TABLE III.

TOTAL NUMBER PATCHES OF E-OPHTA

\begin{tabular}{|l|l|l|l|l|}
\hline Type of Dataset & Microaneurysm & Haemorrhage & Exudate & No-Sign \\
\hline Training & 1376 & N/A & 2517 & 4119 \\
\hline Testing & 918 & N/A & 1678 & 2746 \\
\hline Total Number & 2294 & N/A & 4195 & 6865 \\
\hline
\end{tabular}

\section{CNN - Patch Training}

CNN has been executed using CUDA core NVIDIA GTX980 supported with MATLAB 2017b. The network architecture of $\mathrm{CNN}$ is developed as seen in Fig. 5 using four (4) convolution layers. The first convolution layer using 64 feature maps in each convolution layer is pulled by the kernel size of $(3 \times 3)$ pixels. Next, the activated function uses the rectified liner unit (ReLU) in this research to prevent saturation and allow it to compute faster than the sigmoid [23]. Therefore, the Max-Pooling function helps to reduce the feature map size kernel (2x2) pixel to simplify the output parameter. Thirdly, the Max-Pooling (MP) function is normalised by the normalization layer (NM) to expedite the network training and sensitivity to various networks. Lastly, the Max-Pooling Layer with 512 features was extracted using a fully connected layer (FC) to link with 512 neurons. The output result of FC was normalized by softmax activated function to determine the probabilities classification by the four (4) classification layers.

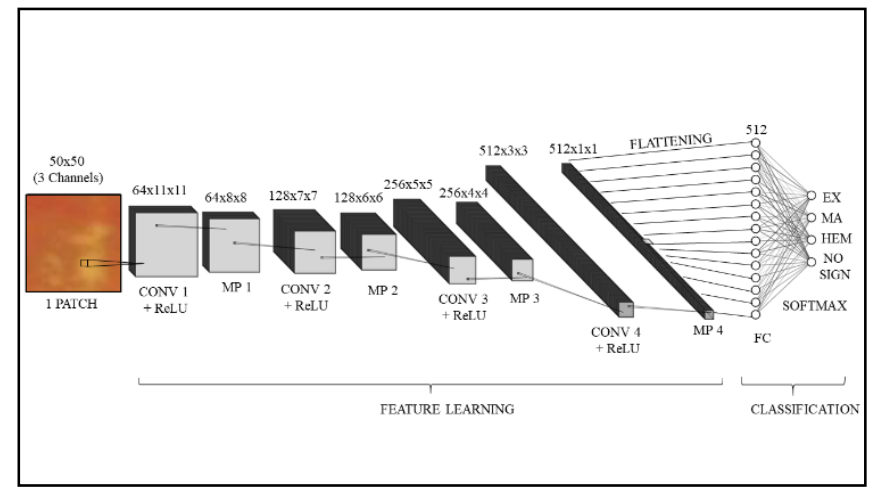

Fig. 5. (CNN) parameter. CONV: convolution layer, MP: max pooling layer, FC: fully connected layer.

To define the value network training parameter on the CNN in MATLAB, the optimum parameter of CNN is defined in Table IV:

TABLE IV.

CNN SETUP INPUT PARAMETER

\begin{tabular}{ll}
\hline CNN parameter & Value \\
\hline Initial Learning Rate & 0.0001 \\
Maximum Epochs & 200 \\
Validation Frequency & 100 iterations \\
Mini Batch Size & 32 \\
Convolution layer & 4 \\
Solver Method & SGDM \\
Filter Size & Step Down $(11,8,7,6,5,4,3,2)$, \\
Feature Map & Step Up $(64,128,256,512)$ \\
Activate Function & ReLU, Softmax \\
\hline
\end{tabular}




\section{Performance Evaluation}

In medical research, the performance of classification is evaluated by using the accuracy and false negative rate (FNR). The accuracy in equation (12) refers to how correct a diagnostic test identifies and excludes a given condition. The false negative rate (13) is used to monitor the type of switching error percentage from the validation. Both performance validation was given as follows:

$$
\begin{aligned}
& \text { Accuracy }=\frac{T P+T N}{T P+T N+F P+F N} \\
& F N R=\frac{F N}{F N+T P}=1-P P V
\end{aligned}
$$

where: $\mathrm{TP}=$ true positive, $\mathrm{TN}=$ true negative, $\mathrm{FP}=$ false positive and $\mathrm{FN}=$ false negative.

\section{RESULTS AND DISCUSSION}

The enhancement method 1 on the retinal image was completely enhanced from the original image by applying the high-pass filter to sharpen the edge and this is followed by the histogram equalization method to increase the contrast of the image. As the result, the output enhance image gives clearly observable pathological signs compared to the original image. The step flow and output enhancement 1 can be observed in Fig. 6.

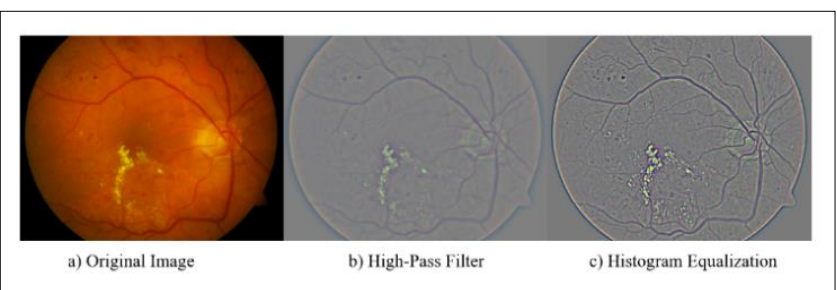

Fig. 6. The output of Histogram Equalization (right) after passing through high pass filter (middle) from the Original image (left).

Similar to the image enhancement method 2 in Fig.7, the image was successfully enhanced by applying high-pass in the first step to remove the noise and sharpen the edge (a). Secondly, the image inverts RGB color from the high pass image (b). Thirdly, the image is applied to de-haze using equation (9) to enhance the low light image as shown in image (d). Finally, the invert operation was reapplied as the resulting enhanced image is able to see more salient pathological signs in (e).

In this experiment, a total of $60 \%$ patch is reserved for training, while the remaining $40 \%$ patches are reserved for the testing and validation purposes. Once CNN starts training, Fig. 7 shows the monitor training progress on the accuracy and error loss of the network against epoch.

During the training progress, the training accuracy and error loss percentage shows improvement significantly starting from early stage until it reached the $9^{\text {th }}$ epoch. However, the accuracy percentage started to slowly converge for more than $90 \%$ while the error started to slowly converge when the error percentage achieved less than $0.3 \%$. This training progress monitoring shows that the CNN model was able to extract and learn the features itself based on the dataset given to classify the pathological signs.

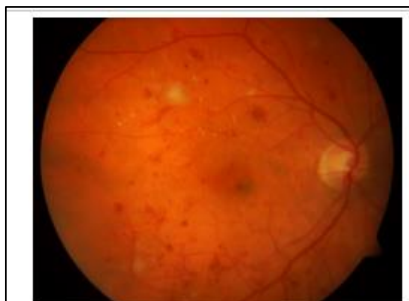

(a)

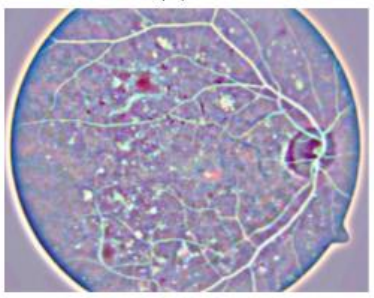

(c)

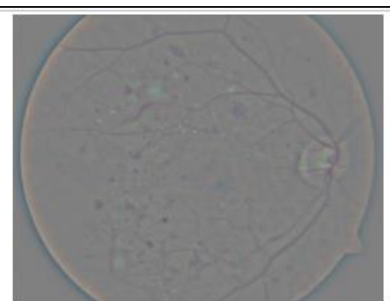

(b)

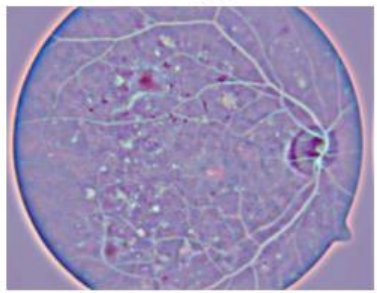

(d)

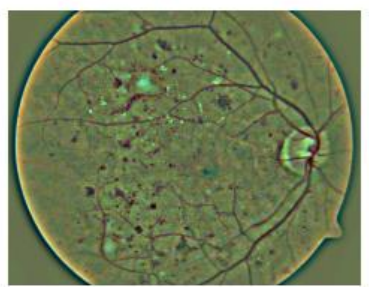

(e)

Fig. 7. (a) Original image, (b) high-pass filter result. (c) Inverted result (d) Applying de-haze algorithm, (e) Final output image reapplying the inverted image algorithm.

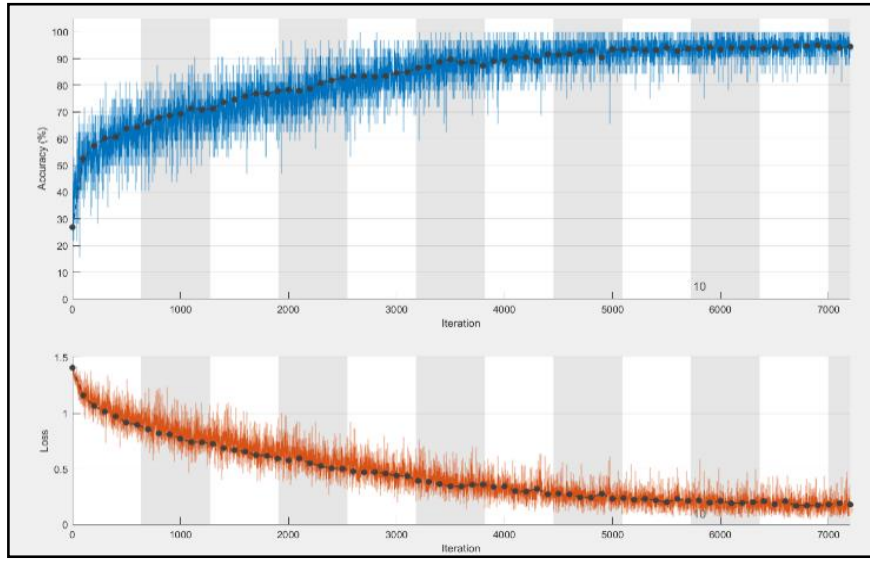

Fig. 7. Deep learning training progress shown accuracy percentage and error loss percentage of the network against iteration.

The CNN performance was validated by using the testing dataset based on the training dataset. The accuracy and error for the false negative rate (FNR) of the $\mathrm{CNN}$ was calculated based on the confusion matrix. The confusion matrix summarizes the result with two dimensions namely the predicted class and actual class. Fig.8, shows an example of the confusion matrix for DIARETDB1 with th eimage enhancement method 1. 


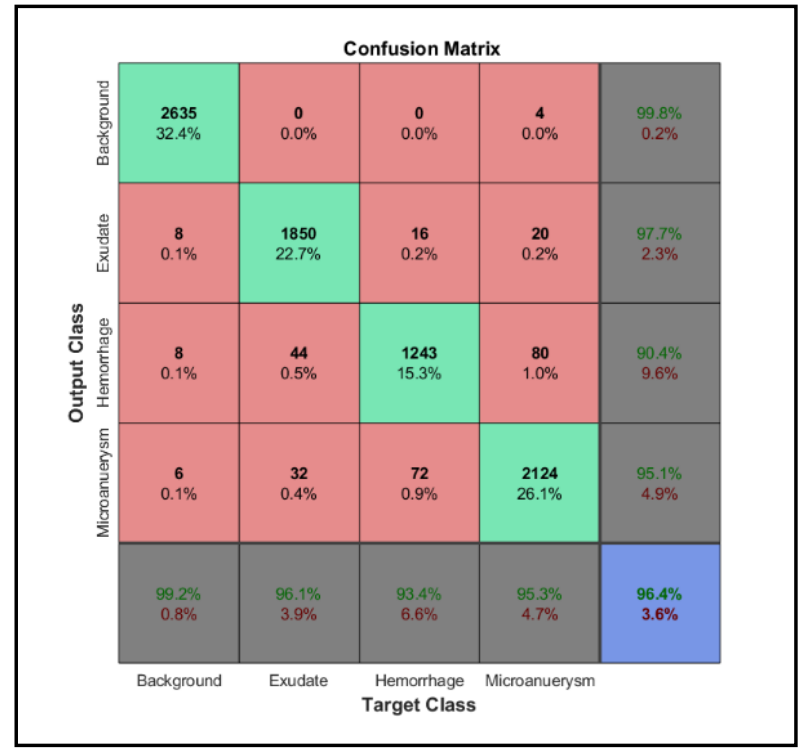

Fig. 8. Total four (4) class of confusion matrix plot for DIARETDB1.

For CNN that optimizes the setup training and validation, we run 4 different layers of convolution with the same 100 epoch at the same dataset enhancement image with the same GPU to optimize the performance of CNN. Variables of Conv Layer are varied using 1 layer(a) 2 layer(b) 3 layer(c) and 4layer(d) as shown in Table V.

From the result in Table V, the increase in the convolution layer will increase the overall performance accuracy of CNN from $94.1 \%$ to $96.3 \%$. The CNN started to achieve the best performance when Conv layer was set as layer 3 which achieved $96.2 \%$ of DIARETDB1 dataset enhancement. However, the increase of Conv layer will increase the training and testing time from $142 \mathrm{~min}$ to $173 \mathrm{~min}$. That said, increasing the Conv layer gives a smooth training accuracy as shown from (a) to (d) as referred to the blue line.

Table VI presents the overall CNN accuracy with the application of the method enhancement and comparison of the performance on the original image. Without the enhancement of the original image, by applying image enchancement method 1 and method 2 it shows that the overall CNN performance accuracy percentage has improved by $4.3 \%$ and $3.6 \%$ respectively for E-Optha. For DIARETDB1, by using the image enhancement method 1 and method 2, it also improved the overall accuracy by $1.2 \%$ and $1.5 \%$ respectively. Both methods of image enhancement resulted in the improved visual quality of pathological signs where they become more visible. As the result, it is easier for the CNN to extract and learn important features which are able to result in better classification of accuracy. From the previous work, many researchers use the image enhancement method 1 as the DR screening. Comparing our result by [8] it is proven that the image enhancement method 2 is a reliable method for DR screening.
TABLE V

CNN CONVOLUTION LAYER ANALYSIS

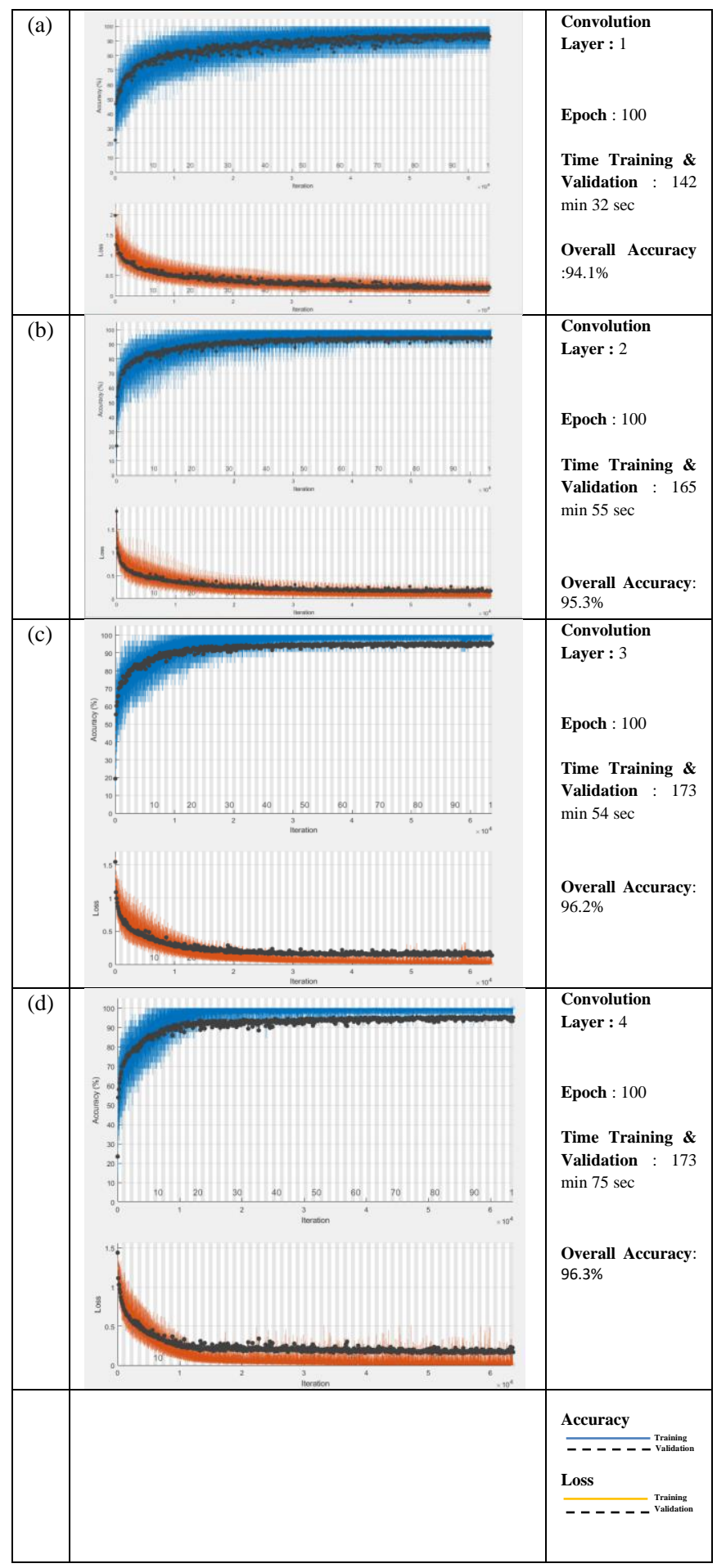


TABLE VI

EFFECT OF PEFORMANCE CNN FOR EACH DATASET DIARETDB1 AND E-OPTHA BY APPLYING ENCHANCEMENT TECHNIQUE

\begin{tabular}{|c|c|c|c|c|c|c|}
\hline \multirow{2}{*}{ Dataset } & \multirow{2}{*}{ Image Pre-Processing } & \multicolumn{5}{|c|}{ Accuracy $(\%)$} \\
\hline & & No Sign & Exudate & MA & HEM & Overall \\
\hline \multirow{3}{*}{ E-Optha } & $\begin{array}{l}\text { Without Enhancement } \\
\text { (Original Image) }\end{array}$ & $\begin{array}{l}97.4 \\
(95.1- \\
99.6)\end{array}$ & $\begin{array}{l}98.8 \\
(97.8- \\
99.4)\end{array}$ & $\begin{array}{l}84.6 \\
(80- \\
92.1)\end{array}$ & N/A & $\begin{array}{l}95.8 \\
(94-97.1)\end{array}$ \\
\hline & $\begin{array}{l}\text { Enhancement } 1 \\
\text { (high Pass + HE) }\end{array}$ & $\begin{array}{l}99.9 \\
(99.9- \\
100)\end{array}$ & $\begin{array}{l}99.7 \\
(99.6- \\
99.7)\end{array}$ & $\begin{array}{l}99.1 \\
(99.2- \\
99.0)\end{array}$ & N/A & $\begin{array}{l}99.8 \\
(99.7-99.8)\end{array}$ \\
\hline & $\begin{array}{l}\text { Enhancement } 2 \\
\text { (high Pass + De-Haze) }\end{array}$ & $\begin{array}{l}99.9(99 . \\
9-100)\end{array}$ & $\begin{array}{l}99.0 \\
(98.9- \\
99.1)\end{array}$ & $\begin{array}{l}99.3 \\
(98.9- \\
99.9)\end{array}$ & N/A & $\begin{array}{l}99.4 \text { (99.3- } \\
99.4)\end{array}$ \\
\hline \multirow{3}{*}{ DIARETDB1 } & $\begin{array}{l}\text { Without Enhancement } \\
\text { (Original Image) }\end{array}$ & $\begin{array}{l}99.1 \\
(98.7- \\
99.3)\end{array}$ & $\begin{array}{l}97.0 \\
(96.6- \\
97.6)\end{array}$ & $\begin{array}{l}88.6 \\
(87.9- \\
89.4)\end{array}$ & $\begin{array}{l}88.5 \\
(85.2- \\
90.4)\end{array}$ & $\begin{array}{l}94.4(93.7- \\
94.8)\end{array}$ \\
\hline & $\begin{array}{l}\text { Enhancement } 1 \\
\text { (high Pass }+\mathrm{HE} \text { ) }\end{array}$ & $\begin{array}{l}99.8 \\
(99.7- \\
99.9)\end{array}$ & $\begin{array}{l}96.2 \\
(95.8- \\
96.6)\end{array}$ & $\begin{array}{l}95.5 \\
(94.2- \\
96.5)\end{array}$ & $\begin{array}{l}990.6 \\
(90.2- \\
91.9)\end{array}$ & $\begin{array}{l}95.6(94.4- \\
96.4)\end{array}$ \\
\hline & $\begin{array}{l}\text { Enhancement } 2 \\
\text { (high Pass + De-Haze) }\end{array}$ & $\begin{array}{l}99.9 \\
(99.8- \\
100)\end{array}$ & $\begin{array}{l}93.9 \\
(93.3- \\
94.3)\end{array}$ & $\begin{array}{l}94.6 \\
(93.9- \\
95.5)\end{array}$ & $\begin{array}{l}91.7 \\
(91.3- \\
92.3)\end{array}$ & $\begin{array}{l}95.9(95.70- \\
95.8)\end{array}$ \\
\hline
\end{tabular}

Even both methods of enhancement resulted in the overall improvement for both datasets, based on the result from Table VI, where there are some limitations and root causes which do not meet the expectation and which makes us to have to take a closer look. The miss predicted confusion matrix is presented as false negative rate (FNR). We have completed an analysis from both datasets E-Optha and DIARETDB1 on the error validation using the false negative rate equation. Both results on FNR are shown in Table VII.

TABLE VII

CNN MISS CLASSFICATION PREDICTION FOR E-OPTHA AND DIARETDB1 WITH AND WITHOUT IMAGE ENCHANCEMENT

\begin{tabular}{|c|c|c|c|c|c|c|}
\hline \multirow{2}{*}{ Dataset } & \multirow{2}{*}{ Image Pre-Processing } & \multicolumn{5}{|c|}{ False Negative Error (\%) } \\
\hline & & No Sign & Exudate & MA & HEM & Overall \\
\hline \multirow{3}{*}{ E-Optha } & $\begin{array}{l}\text { Without Enhancement } \\
\text { (Original Image) }\end{array}$ & $\begin{array}{l}2.6(0.4- \\
4.9)\end{array}$ & $\begin{array}{l}1.2(0.6- \\
2.2)\end{array}$ & $\begin{array}{l}15.4 \\
(7.9-20)\end{array}$ & N/A & $4.2(2.9-6)$ \\
\hline & $\begin{array}{l}\text { Enhancement } 1 \\
\text { (high Pass + HE) }\end{array}$ & $\begin{array}{l}0.1(0- \\
0.1)\end{array}$ & $\begin{array}{l}0.3 \\
(0.3- \\
0.4) \\
\end{array}$ & $\begin{array}{l}0.8 \\
(0.8-1)\end{array}$ & N/A & $0.2(0.2-0.3)$ \\
\hline & $\begin{array}{l}\text { Enhancement } 2 \\
\text { (high Pass + De-Haze) }\end{array}$ & $\begin{array}{l}0.1(0- \\
0.1)\end{array}$ & $\begin{array}{l}1(0.9- \\
1.1)\end{array}$ & $\begin{array}{l}0.7(0.1- \\
1.1)\end{array}$ & N/A & $0.6(0.6-0.7)$ \\
\hline \multirow{3}{*}{ DIARETDB 1} & $\begin{array}{l}\text { Without Enhancement } \\
\text { (Original Image) }\end{array}$ & $\begin{array}{l}0.9(0.7- \\
1.3)\end{array}$ & $\begin{array}{l}0.3 \\
(2.4- \\
3.4) \\
\end{array}$ & $\begin{array}{l}11.4 \\
(10.6- \\
12.1) \\
\end{array}$ & $\begin{array}{l}11.5 \\
(9.6- \\
14.8) \\
\end{array}$ & $5.6(5.2-6.3)$ \\
\hline & $\begin{array}{l}\text { Enhancement } 1 \\
\text { (high Pass + HE) }\end{array}$ & $\begin{array}{l}0.2(0.1- \\
0.3)\end{array}$ & $\begin{array}{l}3.8 \\
(3.4- \\
4.2)\end{array}$ & $\begin{array}{l}4.5(3.5- \\
5.8)\end{array}$ & $\begin{array}{l}9.4(8.1- \\
9.8)\end{array}$ & $4.4(3.6-5.6)$ \\
\hline & $\begin{array}{l}\text { Enhancement } 2 \\
\text { (high Pass + De-Haze) }\end{array}$ & $\begin{array}{l}0.1(0- \\
0.2)\end{array}$ & $\begin{array}{l}6.1 \\
(5.7- \\
6.7)\end{array}$ & $\begin{array}{l}5.4(4.5- \\
6.1) \\
\end{array}$ & $\begin{array}{l}8.3(7.7- \\
8.7) \\
\end{array}$ & $4.1(4.2-4.3)$ \\
\hline
\end{tabular}

First of all, the CNN miss prediction analysis for E-Optha shows that the highest error was on Exudate (0.3-1\%) and MA $(0.7-0.8 \%)$ after applying the image enchancement. The root cause of this miss prediction between Exudate and MA is proven by the image in (b) in Fig.9. This has shown that the same patch had overlapped between Exudate and MA where the CNN appeared uncertain to predict the right classification during both the test and validation.

The second miss prediction analysis on DIARETDB1 dataset shows a major contribution on the error classification that happened on $\operatorname{MA}(4.5-5.4 \%)$ and $\operatorname{HEM}(8.3-9.4 \%)$ after applying image enchancement 1 and 2 . The root cause of the miss prediction is due to the pattern and shape of MA and HEM overlapping in the same patch, which makes CNN confused to classify both signs. In addition HEM and MA have a similarity in terms of the morphological shape. These can be proven in (a) Fig.9. In general, CNN had achieved higher accuracy for EOptha dataset because the category is lesser to classify compared to DIARETDB1 [23]. Lasty, the patch with MA exists nearly with no-sign that contains blood vessel making CNN left with slight error to identfy MA or background (nosign). This can be observed in Fig. 9 (c).

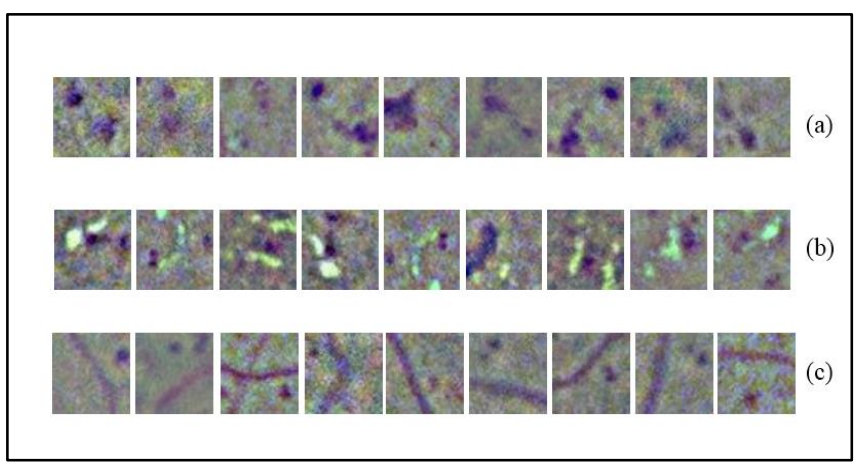

Fig. 9. (a) Microaneurysm and Hemorrhage overlap in the same patch. (b) Microaneurysm and Exudate overlap in the same patch. (c) The No-Sign patches contain vessels that overlap with Microaneurysm.

\section{CONCLUSION}

Overall, this paper has focused on the developed deep learning algorithms using a convolution neural network (CNN) for the automated detection of Diabetic Retinopathy (DR) signs in fundus images. In other words, the idea to propose de-haze and histogram equalization technique on the image enhancement helps offer better overall accuracy. The result of comparison with and without the enhancement classification accuracy for DIARETDB1 improved $1.2 \%$ and $1.5 \%$ for enhancement 1 and 2 respectively, while e-Ophtha has increased the accuracy by $4.3 \%$ and $3.6 \%$ supported by enhancement 1 and 2 respectively. In future, other improvement methods need to be investigated to improve the overall accuracy of the compilation test with different pathological signs. Future work will also add more new datasets from local hospitals.

\section{ACKNOWLEDGMENT}

Author would like to acknowledge Universiti Teknologi MARA (UiTM) especially the Faculty of Electrical Engineering for providing financial allocation on this research and the Faculty of Medicine (Ophthalmology department) for the collaboration effort in this work.

\section{REFERENCES}

[1] D. M. Squirrell and J. F. Talbot, "Screening of Diabetic Retinopathy," J. R. Soc. Med., vol. 96, no. 6, pp. 273-276, 2011, doi: 10.1002/pdi.299.

[2] R. S. Biyani and B. M. Patre, "Algorithms for red lesion detection in Diabetic Retinopathy: A review," Biomed. Pharmacother., vol. 107, no. July, pp. 681-688, 2018, doi: 10.1016/j.biopha.2018.07.175.

[3] Ministry of Health Malaysia, "Diabetic retinopathy screening: Training module for healthcare providers," p. 88, 2017, doi: 10.1038/eye.2001.40.

[4] J. I. Orlando, E. Prokofyeva, M. del Fresno, and M. B. Blaschko, "An ensemble deep learning based approach for red lesion detection in fundus images," Comput. Methods Programs Biomed., vol. 153, pp. 115-127, 2018, doi: 10.1016/j.cmpb.2017.10.017.

[5] G. G. Gardner, D. Keating, T. H. Williamson, and A. T. Elliott, "Automatic detection of diabetic retinopathy using an artificial neural network: A screening tool," Br. J. Ophthalmol., vol. 80, no. 11, pp. 940- 
944, 1996, doi: 10.1136/bjo.80.11.940.

[6] K. Noronha, U. R. Acharya, K. P. Nayak, S. Kamath, and S. V. Bhandary, "Decision support system for diabetic retinopathy using discrete wavelet transform," Proc. Inst. Mech. Eng. Part H J. Eng. Med., vol. 227, no. 3, pp. 251-261, 2013, doi: 10.1177/0954411912470240.

[7] B. Dupas et al., "Evaluation of automated fundus photograph analysis algorithms for detecting microaneurysms, haemorrhages and exudates, and of a computer-assisted diagnostic system for grading diabetic retinopathy," Diabetes Metab., vol. 36, no. 3, pp. 213-220, 2010, doi: 10.1016/j.diabet.2010.01.002.

[8] P. Khojasteh, B. Aliahmad, and D. K. Kumar, "Fundus images analysis using deep features for detection of exudates, hemorrhages and microaneurysms," BMC Ophthalmol., vol. 18, no. 1, pp. 1-13, 2018, doi: 10.1186/s12886-018-0954-4.

[9] P. Chudzik, S. Majumdar, F. Calivá, B. Al-Diri, and A. Hunter, "Microaneurysm detection using fully convolutional neural networks," Comput. Methods Programs Biomed., vol. 158, pp. 185-192, 2018, doi: 10.1016/j.cmpb.2018.02.016.

[10] L. Dai et al., "Clinical report guided retinal microaneurysm detection with multi-sieving deep learning," IEEE Trans. Med. Imaging, vol. 37, no. 5, pp. 1149-1161, 2018, doi: 10.1109/TMI.2018.2794988.

[11] P. N. S. Kumar, R. U. Deepak, A. Sathar, V. Sahasranamam, and R. R. Kumar, "Automated Detection System for Diabetic Retinopathy Using Two Field Fundus Photography," Procedia Comput. Sci., vol. 93, no. September, pp. 486-494, 2016, doi: 10.1016/j.procs.2016.07.237.

[12] M. R. K. Mookiah, U. R. Acharya, C. K. Chua, C. M. Lim, E. Y. K. Ng, and A. Laude, "Computer-aided diagnosis of diabetic retinopathy: A review," Comput. Biol. Med., vol. 43, no. 12, pp. 2136-2155, 2013, doi: 10.1016/j.compbiomed.2013.10.007.

[13] R. F. Mansour, "Evolutionary Computing Enriched Computer-Aided Diagnosis System for Diabetic Retinopathy: A Survey," IEEE Rev. Biomed. Eng., vol. 10, pp. 334-349, 2017, doi: 10.1109/RBME.2017.2705064.

[14] E. Decencière et al., "TeleOphta: Machine learning and image processing methods for teleophthalmology," Irbm, vol. 34, no. 2, pp. 196-203, 2013, doi: 10.1016/j.irbm.2013.01.010.

[15] A. Dogra and P. Bhalla, "Image sharpening by Gaussian and Butterworth high pass filter,” Biomed. Pharmacol. J., vol. 7, no. 2, pp. 707-713, 2014, doi: $10.13005 / \mathrm{bpj} / 545$.

[16] M. Ahmad, N. Zaman, and M. Al-Amin, "An experimental research in health informatics for enhancing ovarian cancer identification in ovarian imaging analysis using fuzzy histogram equalization," J. Med. Imaging Heal. Informatics, vol. 7, no. 6, pp. 1385-1390, 2017.

[17] R. Abbasi, Z. Wang, and B. Luo, "Dynamic weighted histogram equalization for contrast enhancement using for Cancer Progression Detection in medical imaging," no. 1, pp. 3-8.

[18] W.-Y. Hsu and C.-Y. Chou, "Medical image enhancement using modified color histogram equalization," J. Med. Biol. Eng., vol. 35, no. 5, pp. 580-584, 2015.

[19] D. J. Hemanth, O. Deperlioglu, and U. Kose, "An enhanced diabetic retinopathy detection and classification approach using deep convolutional neural network," Neural Comput. Appl., vol. 32, no. 3, pp. 707-721, 2020, doi: 10.1007/s00521-018-03974-0.

[20] O. Deperlioglu, U. Kose, and G. Emre Guraksin, "Underwater Image Enhancement with HSV and Histogram Equalization," Int. Conf. Adv. Technol. (ICAT 2018), 2018.

[21] X. Dong et al., "Fast efficient algorithm for enhancement of low lighting video," Proc. - IEEE Int. Conf. Multimed. Expo, vol. 3, pp. 3-8, 2011, doi: 10.1109/ICME.2011.6012107.

[22] H. Kaiming, S. Jian, and T. Xiaoou, "Single image haze removal using dark channel prior. Single image haze removal using dark channel prior.," Cvpr, vol. 33, no. 12, pp. 2341-2353, 2009, [Online]. Available: http://kaiminghe.com/publications/cvpr09.pdf.

[23] A. H. Abu Samah, F. Ahmad, M. K. Osman, M. Idris, N. M. Tahir, and N. A. Abd. Aziz, "Classification of Pathological Signs for Diabetic Retinopathy Diagnosis using Image Enhancement Technique and Convolution Neural Network," 2019 9th IEEE Int. Conf. Control Syst. Comput. Eng., pp. 221-225, 2020, doi: 10.1109/iccsce47578.2019.9068538.

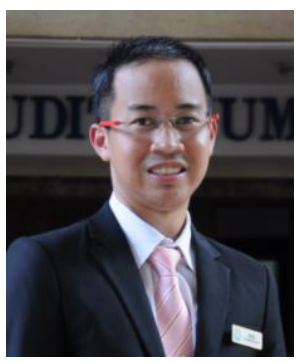

Abdul Hafiz Abu Samah received his first B.Eng degree in Electrical and Electronic Engineering from Universti Teknologi MARA in 2018. He currently serve as Administrative and Diplomatic Officer (PTD) in Secretary of State office in Negeri Sembilan. He has gained experience in industries as he serve as Product Development Engineer at Intel Technologies from 2018 to 2020 . He currently is doing his Master Degree at Universtiti Teknologi MARA in research. Hi research focus on the image processing of medical image, specifically in analysing DR image using deep learning.

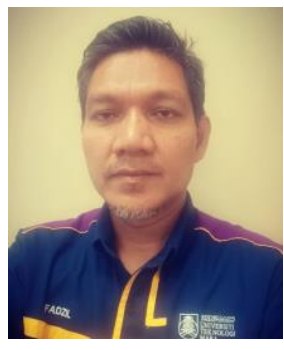

Fadzil Ahmad obtained his Ph.D degree in computational intelligence and Bachelor degree in Electrical and Electronic Engineering from the School of Electrical and Electronics Engineering, Universiti Sains Malaysia in 1997 and 2016 respectively. He is currently an academician at Faculty of Electrical Engineering, Universiti Teknologi MARA Pulau Pinang, Malaysia since 2004. He has gained vast industrial experience in the electronics sector as he served Agilent Technologies and Advanced Micro Devices as a test engineer from 1997 to 2002 . His primary research interests include evolutionary algorithm, image processing and machine learning with applications to intelligent medical decision and diagnosis, pattern recognition, classification and optimization.

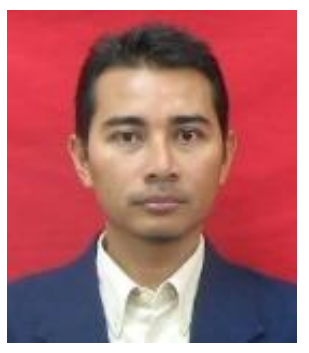

Muhammad Khusairi Osman obtained his B.Eng degree in Electrical and Electronic Engineering in 2000 and MSc in Electrical and Electronic Engineering in 2004 from Universiti Sains Malaysia. In 2014, he obtained his Ph.D. in medical electronic engineering from Universiti Malaysia Perlis (UniMAP), Malaysia. He is currently a senior lecturer at Faculty of Electrical Engineering, Universiti Teknologi MARA (UiTM), Malaysia. His research interest is in image processing, pattern recognition and artificial intelligence.

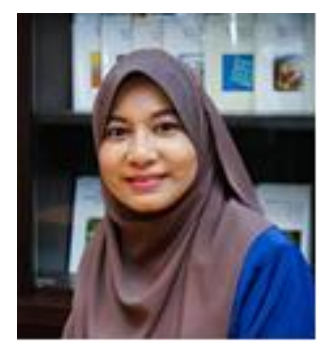

Nooritawati Md Tahir is a lecturer at the Faculty of Electrical Engineering, Universiti Teknologi MARA Selangor Malaysia. Her research interest is in the field of Image Processing, Pattern Recognition and Computational Intelligence. She has authored and co-authored more than 250 indexed publications with SCOPUS h-index of 18. In addition, she is a Certified Trainer for Postgraduate Training in Innovation and Entrepreneurship awarded by TCD-UCD Dublin, Ireland, Stanford Faculty Fellow organised by Stanford Technology Ventures Program, Stanford University, US and Chartered Engineer (CEng) from the Institution of Engineering and Technology and Engineering 
Council UK as well as Senior Member (SMIEEE) of Institution of Electrical, Electronic Engineer (IEEE).

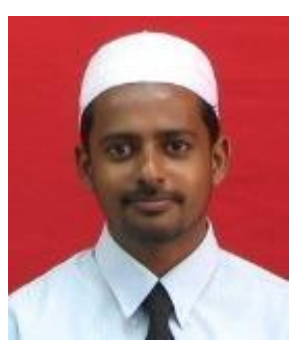

Mohaiyedin Idris obtained his Diploma in Electronic Engineering (Communication) in 2000 from Politeknik Ungku Omar. In 2005 his obtained B.Eng degree in Electrical Electronic Engineering from Universiti Teknologi Malaysia and then MSc in Electrical and Electronic Engineering in 2010 from Universiti Sains Malaysia. $\mathrm{He}$ is currently a senior lecturer at Faculty of Electrical Engineering, Universiti Teknologi MARA (UiTM), Malaysia. His research interest is in image processing, pattern recognition, artificial intelligence, electronic system design, software design and database design.

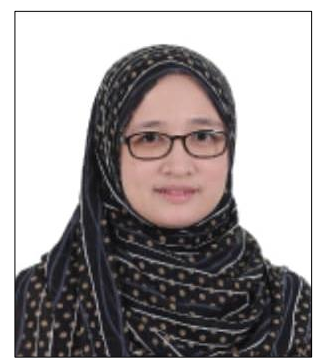

Dr Nor Azimah binti Abd Aziz obtained her basic degree in Medicine in year 2004. She pursued postgraduate training in ophthalmology and obtained Master Degree of Medicine (Ophthalmology) in 2015. She is currently a General Ophthalmologist and Senior Lecturer at Faculty of Medicine, Universiti Teknologi MARA (UiTM), Malaysia. Her research interest is in medical retina, public health ophthalmology and innovative teaching and learning 